\title{
Synthesis, Cytotoxicity Assessment and Molecular Docking of N-(5-(substituted-benzylthio)-1,3,4- thiadiazole-2-yl)-2-p-fluorophenylacetamide Derivatives as Tyrosine Kinase Inhibitors
}

\author{
Y. BAHMANI, T. BAHRAMI ${ }^{1}$, AND A. ALABADI $12^{1 *}$ \\ Students Research Committee, ${ }^{1}$ Phamaceutical Sciences Research Center, School of Pharmacy, ${ }^{2}$ Department of Medicinal \\ Chemistry, Faculty of Pharmacy, Kermanshah University of Medical Sciences, Kermanshah, Iran
}

Bahmani et al.: 1,3,4-Thiadiazoles as Potential Anticancer Agents

\begin{abstract}
Compounds containing 1,3,4-thiadiazole nucleus appear to be potential tyrosine kinase inhibitors. Previous reports showed that some 1,3,4-thiadiazole derivatives were designed as probable tyrosine kinase inhibitors. Thiol derivative (2) was obtained from the reaction of 5-amino-1,3,4-thiadiazole-2-thiol with 4-fluorophenylacetic acid, ethyldimethyaminopropylcarbodiimide and hydroxybenzotriazole. Subsequent reaction of the obtained thiol derivative with diverse benzyl chlorides afforded the final compounds 3a-3l in a click reaction surprisingly. Derivatives with electron withdrawing moieties $(\mathrm{F}, \mathrm{Cl})$ exerted higher yield compared to methoxylated derivatives as electron donating group. Besides, docking studies using ArgusLab 4.0 was done for exploring the probable binding mode and interactions. Investigation of cytotoxicity of target compounds (3a-3l) by MTT assay revealed that these derivatives are more active against the breast cancer cell line MCF-7 and most of these were found to be more effective than imatinib as reference drug. Chlorine containing derivatives at ortho and meta positions were the most cytotoxic in these series.
\end{abstract}

Key words: Synthesis, 1,3,4-thiadiazole, cytotoxicity, tyrosine kinase, docking, anticancer

Cancer as neoplastic disorder is a collection of diseases in which cells can have various properties such as aggressive, invasive and/or metastasizing. These three malignant properties of cancer are their differentiative parts from benign tumours, which are self-limited in their growth and do not invade or metastasize ${ }^{[1]}$. Despite high advances in diagnosis and treatment of neoplastic diseases, overall survival and cure of patients still remains poor. Until recent years, surgery, chemotherapy, radiotherapy, and hormonal therapy have been the standard and routine therapeutic options available for patients. This has improved and supported survival in several types of solid tumours (breast cancer, prostate carcinoma) but drug toxicity related to chemotherapy and emergence of drug resistance in tumours has been the major cause of morbidity and mortality.

Rapid scientific advances in cell biology in recently have enhanced our comprehension of the molecular mechanism of cancer and consequently increased the capability of scientists for anticancer drug design and discovery ${ }^{[2]}$. Targeted therapies like inhibition of overexpressed receptor/enzymes in some tumours have become one of the latest innovative trends in the treatment of cancer. Attempt to inhibit a specific molecular target that is hypothesized to have a pivotal role in a growth or progression of a specific tumor is a logical and so effective strategy for combating cancer ${ }^{[3]}$. Tyrosine kinases have been recognized as a new promising molecular target for treatment of cancer. Cell division and differentiation are important phenomenon in cells that regulate by signal transduction associated with receptor tyrosine kinases ${ }^{[2,4]}$. Incidence of some types of cancer is associated with mutation in genes

This is an open access article distributed under the terms of the Creative Commons Attribution-NonCommercial-ShareAlike 3.0 License, which allows others to remix, tweak, and build upon the work non-commercially, as long as the author is credited and the new creations are licensed under the identical terms

Accepted 21 November 2018

Revised 20 April 2018

Received 24 April 2017

Indian J Pharm Sci 2019;81(1):63-70 
responsible for tyrosine kinase encoding ${ }^{[5]}$. Ninety-five percent of all chronic myelogenous leukaemia was suggested to be result of the altered tyrosine kinase like $\mathrm{Abl}$ that, under physiological conditions, is under tight control but in fusion is deregulated and expressed constitutively resulting in indefinite proliferation ${ }^{[6]}$. The role of c-Src tyrosine kinase is also verified in some solid tumours like breast cancer ${ }^{[7]}$. Imatinib and dasatinib are new anticancer drugs with mechanism related to the inhibition of tyrosine kinases (fig. 1 $)^{[8]}$.

Some heterocyclic rings such as 1,3,4-thiadiazole are very attractive research area in medicinal chemistry. In recent years, vast reports about the biological and pharmacological effects of this heterocyclic structure have been provided by scientists. The related pharmacological activities of 1,3,4-thiadiazole derivatives are antibacterial, antifungal, anticonvulsant, antitubercular, antiinflammatory and anticancer ${ }^{[9-12]}$. Some popular medications in the clinics like cefalothin (an antibiotic) and acetazolamide (diuretic) have 1,3,4-thiadiazole in their pharmacophore. Potential of anticancer activity of this structure is one the most interesting areas that has been encouraged researchers to focus on it. Hence, a large numbers of 1,3,4-thiadiazole derivatives have been synthesized and their molecular mechanism of anticancer activity have been investigated. Inhibition of tyrosine kinases and induction of cellular apoptosis are the most prevalence molecular mechanisms for anticancer effectiveness of 1,3,4-thiadiazole-based compounds ${ }^{[13-17]}$. Based on the above information as well as our previous studies $^{[18-20]}$, we decided to synthesize a new series of 1,3,4-thiadiazole-based compounds and assess their anticancer potency in vitro. Moreover, molecular docking study on two types of cancer related tyrosine kinases namely $\mathrm{Abl}$ and $\mathrm{Src}$ tyrosine kinases was also carried out and their binding modes and interactions were compared to imatinib and dasatinib.

\section{MATERIALS AND METHODS}

All starting materials, reagents and solvents were purchased from commercial suppliers like Merck and Aldrich companies. The purity of the prepared compounds was proved by thin-layer chromatography (TLC) using various solvents of different polarities. Merck silica gel $60 \mathrm{~F}_{254}$ plates were applied for analytical TLC. Column chromatography was performed on Merck silica gel (70-230 mesh) for purification of intermediate and final compounds. ${ }^{1} \mathrm{H}$-nuclear magnetic resonance ( $\left.{ }^{1} \mathrm{HNMR}\right)$ spectra were

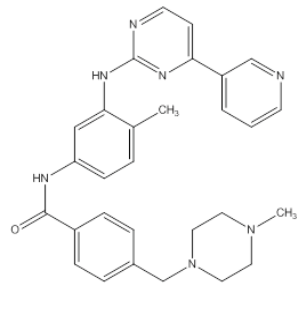

A

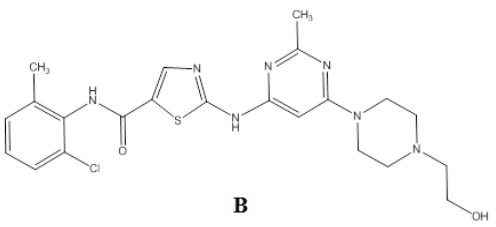

Fig. 1: Structures of two clinically used tyrosine kinase inhibitors A: Imatinib; B: dasatinib

recorded using a Varian 400 spectrometer, and chemical shifts are expressed as $\delta(\mathrm{ppm})$ with tetramethylsilane as the internal standard. The IR spectra were obtained on a Shimadzu 470 spectrophotometer (potassium bromide disks). Melting points (MP) were determined using electrothermal melting point analyser apparatus and are uncorrected. The mass spectra were run on a Finnigan TSQ-70 spectrometer (Finnigan, USA) at $70 \mathrm{eV}$. All cell lines were purchased from Pasteur Institute of Iran.

\section{Chemistry:}

According to the fig. 2, 5-amino-1,3,4-thiadiazole2-thiol (1) was treated directly with 4-fluorophenylacetic acid for amide bond formation. The reaction was carried out in the presence of ethyl dimethyaminopropylcarbodimide and hydroxybenzotriazole in acetonitrile as solvent. The completion of reaction was confirmed by TLC. After completion, the solvent was evaporated under reduced pressure and ethyl acetate/water was added. The aqueous phase was separated and the organic phase was washed two times by sodium bicarbonate $10 \%$, diluted sulphuric acid and brine. Anhydrous sodium sulphate was added and filtration was done. Ethyl acetate was removed using rotatory evaporator and a yellow powder was obtained. The obtained product was used after crystallization from ethanol for the next step. Various benzyl chloride derivatives were reacted with compound 2 for obtaining the final appropriate products (3a-31). NMR, IR and mass spectra (MS) were used to confirm the synthesized compounds.

Compound 2 were synthesized according to our previous literature ${ }^{[19]}$. Compounds $3 a-31$ was synthesized by reacting equimolar quantities of each benzyl chloride derivative with 2-(4-fluorophenyl)- $\mathrm{N}$ (5-mercapto-1,3,4-thiadiazol-2-yl)acetamide (2). At first, compound 2 was converted to a thiolate anion using equimolar potassium hydroxide in absolute 

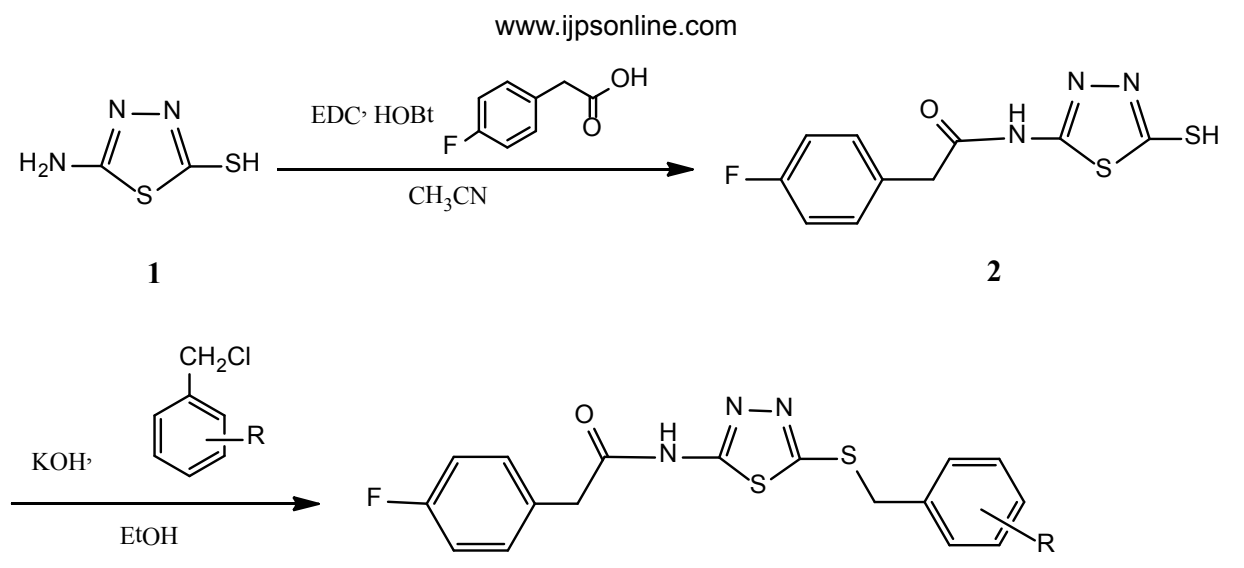

$\mathrm{R}: \mathrm{NO}_{2},{ }^{-} \mathrm{OCH}_{3}, \mathrm{Cl}, \mathrm{F}, \mathrm{H} \quad \mathbf{3 a - 3 l}$

Fig. 2: Synthetic procedure for synthesis of compounds 3a-3I

ethanol for accelerating the nucleophillic attack. Then, appropriate benzyl chloride derivative was added to the reaction medium. Only with hand shaking in few seconds, the intended products (3a-31) were formed and precipitated in the reaction medium (a click reaction occurred) surprisingly except compound $31(24 \mathrm{~h}$ reflux condition was needed for this derivative). The precipitate was cooled by crushed ice, filtered, washed by cold water and purified by appropriate procedures such as crystallization from ethanol or column chromatography (EtOAC/petroleum ether: 3/2) $)^{[18,21]}$.

N-(5-(2-fluorobenzylthio)-1,3,4-thiadiazol-2-yl)-2(4-fluorophenyl)acetamide (3a); MP: $195^{\circ}$, yield: 79\%, ${ }^{1} \mathrm{HNMR}$ (DMSO-d $6,400 \mathrm{MHz}$ ) $\delta: 3.8$ (s, 2H, $-\mathrm{CH}_{2} \mathrm{CO}-$ ), $4.48\left(\mathrm{~s}, 2 \mathrm{H},-\mathrm{CH}_{2} \mathrm{~S}-\right), 7.04$ (m, 4H, 2-fluorobenzyl), 7.25 (m, 2H, $\mathrm{H}_{2,6}$-4-fluorophenyl), 7.35 (m, 2H, $\mathrm{H}_{3,5}$ 4-fluorophenyl), 12.6 (brs, NH). IR (KBr, $\left.\mathrm{cm}^{-1}\right): 3442$ (stretch, N-H), 3158 (C-H, aromatic), 2856 (stretch, C-H), 1697 (stretch, C=O), 1564 (bend, N-H), 1510, 1489, 1299, 1216, 1157, 840, 758. MS ( $\mathrm{m} / \mathrm{z}, \%): 377$ $\left(\mathrm{M}^{+}, 65\right), 166$ (50), 121 (60), 109 (100), 83 (45), 69 (55), 57 (60), 43 (15).

N-(5-(3-fluorobenzylthio)-1,3,4-thiadiazol-2-yl)-2(4-fluorophenyl)acetamide (3b); MP: $171^{\circ}$, yield: $57 \%$, ${ }^{1} \mathrm{HNMR}\left(\mathrm{CDCl}_{3}, 400 \mathrm{MHz}\right) \delta: 3.97$ (s, 2H, - $\left.\mathrm{CH}_{2} \mathrm{CO}-\right)$, 4.43 (s, 2H, - $\left.\mathrm{CH}_{2} \mathrm{~S}-\right), 7.02$ (m, 4H, 3-fluorophenyl), 7.37 (m, 4H, 4-fluorophenyl), 12.63 (brs, NH). IR $\left(\mathrm{KBr}, \mathrm{cm}^{-1}\right) \bar{v}: 3448$ (stretch, N-H), 3158 (stretch, C-H, aromatic), 2852 (stretch, C-H, aliphatic), 1687 (stretch, $\mathrm{C}=\mathrm{O}$ ), 1566 (bend, N-H), 1511, 1402, 1304, 1230, 1178, 831, 787. MS (m/z, \%): $377\left(\mathrm{M}^{+}, 50\right), 166(90)$, 121 (65), 109 (100), 83 (35), 69 (45), 57 (52), 43 (55).

N-(5-(4-fluorobenzylthio)-1,3,4-thiadiazol-2-yl)-2(4-fluorophenyl)acetamide (3c); MP: 184일 yield: $80 \%$,
${ }^{1} \mathrm{HNMR}\left(\mathrm{CDCl}_{3}, 400 \mathrm{MHz}\right) \delta: 3.98$ (s, 2H, $\left.-\mathrm{CH}_{2} \mathrm{CO}-\right)$, $4.41\left(\mathrm{~s}, 2 \mathrm{H},-\mathrm{CH}_{2} \mathrm{~S}-\right), 6.95\left(\mathrm{t}, 2 \mathrm{H}, J=8 \mathrm{~Hz}, \mathrm{H}_{2,6}\right.$ 4-fluorophenyl), $7.03 \quad\left(\mathrm{t}, \quad 2 \mathrm{H}, \quad J=8 \mathrm{~Hz}, \quad \mathrm{H}_{3,5}-4-\right.$ fluorophenyl), $7.33\left(\mathrm{t}, \quad 2 \mathrm{H}, \quad J=8 \mathrm{~Hz}, \quad \mathrm{H}_{2,6}-4-\right.$ fluorobenzylthio), 7.39 (t, $2 \mathrm{H}, \quad J=8 \mathrm{~Hz}, \quad \mathrm{H}_{3,5}$ 4-fluorobenzylthio), 12.6 (brs, NH). IR ( $\left.\mathrm{KBr}, \mathrm{cm}^{-1}\right)$ v: 3142 (stretch, N-H), 3097 (stretch, C-H, aromatic), 2924 (stretch, C-H, aliphatic), 1693 (stretch, $\mathrm{C}=\mathrm{O}$ ), 1568 (bend, N-H), 1508, 1300, 1222, 1170, 1080, 960, 780, 740. MS (m/z, \%): $377\left(\mathrm{M}^{+}, 80\right), 166(80), 121$ (52), 109 (100), 83 (85), 69 (67), 57 (52), 43 (35).

N-(5-(2-chlorobenzylthio)-1,3,4-thiadiazol-2-yl)2-(4-fluorophenyl)acetamide (3d); MP: 215-218 , yield: $75 \%,{ }^{1} \mathrm{HNMR}\left(\mathrm{DMSO}-\mathrm{d}_{6}, 400 \mathrm{MHz}\right) \delta: 3.79$ (s, $\left.2 \mathrm{H},-\mathrm{CH}_{2} \mathrm{CO}-\right), 4.55\left(\mathrm{~s}, 2 \mathrm{H},-\mathrm{CH}_{2} \mathrm{~S}-\right), 7.01\left(\mathrm{t}, 2 \mathrm{H}, \mathrm{H}_{2,6}-4-\right.$ fluorophenyl), $7.22\left(\mathrm{~m}, 2 \mathrm{H}, \mathrm{H}_{4}, \mathrm{H}_{6}\right.$-2-chlorobenzylthio), 7.33 (t, 2H, $J=8 \mathrm{~Hz}, \mathrm{H}_{3,5}$-4-fluorophenyl), 7.37-7.44 (m, 2H, $\mathrm{H}_{3}, \mathrm{H}_{5}$-2-chlorobenzylthio), 12.6 (brs, NH). IR $\left(\mathrm{KBr}, \mathrm{cm}^{-1}\right) \bar{v}: 3158$ (stretch, N-H), 2853 (stretch, C-H, aliphatic), 2725, 1697 (stretch, $\mathrm{C}=\mathrm{O}$ ), 1565 (bend, $\mathrm{N}-\mathrm{H}), 1509,1443,1413,1351,1298,1217,1158$, 1053, 963, 840, 759. MS (m/z, \%): $394\left(\mathrm{M}^{+1}, 100\right), 393$ $\left(\mathrm{M}^{+}, 85\right), 295$ (45), 182 (30), 125 (35), 109 (60).

N-(5-(3-chlorobenzylthio)-1,3,4-thiadiazol-2-yl)-2-(4fluorophenyl)acetamide (3e); MP: $190^{\circ}$, yield: $51 \%$, ${ }^{1} \mathrm{HNMR}\left(\mathrm{CDCl}_{3}, 400 \mathrm{MHz}\right) \delta: 3.96$ (s, 2H, $\left.-\mathrm{CH}_{2} \mathrm{CO}-\right)$, 4.41 (s, 2H, - $\left.\mathrm{CH}_{2} \mathrm{~S}-\right)$, 6.95 (t, 2H, $\mathrm{H}_{2,6}$-4-fluorophenyl), 7.02 (t, $J=8 \mathrm{~Hz}, \mathrm{H}_{3,5}$-4-fluorophenyl), $7.22\left(\mathrm{~m}, 1 \mathrm{H}, \mathrm{H}_{5}-3\right.$ chlorobenzyl), $7.36\left(\mathrm{~m}, 3 \mathrm{H}, \mathrm{H}_{2}, \mathrm{H}_{4}, \mathrm{H}_{6}\right.$-3-chlorobenzyl), 12.6 (brs, NH). IR (KBr, cm ${ }^{-1}$ ) ṽ: 3425 (stretch, N-H), 3136 (stretch, C-H, aromatic), 2880 (stretch, C-H, aliphatic), 1692 (stretch, $\mathrm{C}=\mathrm{O}$ ), 1547 (bend, N-H), 1508, 1354, 1300, 1214, 1154, 1084, 960, 831, 785. MS (m/z, \%): $394\left(\mathrm{M}^{+1}, 100\right), 393\left(\mathrm{M}^{+}, 65\right), 295$ (55), 182 (25), 125 (45), 109 (30). 
N-(5-(4-chlorobenzylthio)-1,3,4-thiadiazol-2-yl)-2(4-fluorophenyl)acetamide (3f); MP: $187^{\circ}$, yield: $68 \%,{ }^{1} \mathrm{HNMR}$ (DMSO-d, $\left.400 \mathrm{MHz}\right) \quad \delta: 3.97$ (s, $\left.2 \mathrm{H},-\mathrm{CH}_{2} \mathrm{CO}-\right), 4.4\left(\mathrm{~s}, 2 \mathrm{H},-\mathrm{CH}_{2} \mathrm{~S}-\right), 7.03(\mathrm{t}, 2 \mathrm{H}$, $J=8 \mathrm{~Hz}, \mathrm{H}_{2,6}$-4-fluorophenyl), 7.24-7.27 (m, 4H, $\mathrm{H}_{2,3,4,6}$-4-chlorobenzyl), 7.37 ( $\mathrm{t}, 2 \mathrm{H}, J=8 \mathrm{~Hz}, \mathrm{H}_{3,5}-4-$ fluorophenyl), 12.62 (brs, NH). IR (KBr, cm $\left.{ }^{-1}\right) \bar{v}: 3142$ (stretch, N-H), 3118 (stretch, C-H, aromatic), 1693 (stretch, C=O), 1568 (bend, N-H), 1508, 1290, 1222, 1330, 1080, 780. MS (m/z, \%): $394\left(\mathrm{M}^{+1}, 100\right), 393$ $\left(\mathrm{M}^{+}, 80\right), 295$ (35), 182 (40), 125 (75), 109 (60).

2-(4-Fluorophenyl)-N-(5-(2-nitrobenzylthio)-1,3, 4-thiadiazol-2-yl)acetamide (3g); MP: $148-150^{\circ}$, yield: $46 \%,{ }^{1} \mathrm{HNMR}$ (DMSO-d, $400 \mathrm{MHz}$ ) $\delta: 3.68$ (s, $\left.2 \mathrm{H},-\mathrm{CH}_{2} \mathrm{CO}-\right), 4.68$ (s, 2H, $\left.-\mathrm{CH}_{2} \mathrm{~S}-\right), 6.90$ (t, 2H, J=8 $\mathrm{Hz}, \mathrm{H}_{2,6}$-4-fluorophenyl), 7.21 ( $\mathrm{t}, 2 \mathrm{H}, \mathrm{J}=8 \mathrm{~Hz}, \mathrm{H}_{3,5}-4-$ fluorophenyl), 7.36-7.5 (m, $2 \mathrm{H}, \mathrm{H}_{4}, \mathrm{H}_{6}$-2-nitrobenzyl), $7.94\left(\mathrm{~d}, 2 \mathrm{H}, \mathrm{J}=8 \mathrm{~Hz}, \mathrm{H}_{3}, \mathrm{H}_{5}\right.$-2-nitrobenzyl), 12.55 (brs, NH). IR (KBr, cm ${ }^{-1}$ ): 3446 (stretch, N-H), 2920 (stretch, C-H, aromatic), 1698 (stretch, $\mathrm{C}=\mathrm{O}$ ), 1573 (bend, N-H), 1525, 1510, 1336, 1315, 1223, 1135, 1064, 790. MS (m/z, \%): $404(\mathrm{M}+, 15), 149$ (30), 145 (70), 136 (35), 109 (100), 97 (85), 83 (25), 69 (40), 55 (60), 43 (25).

2-(4-Fluorophenyl)-N-(5-(3-nitrobenzylthio)-1,3,4thiadiazol-2-yl)acetamide (3h); MP: $187-190^{\circ}$, yield: $33 \%,{ }^{1} \mathrm{H}$ NMR (DMSO-d, $400 \mathrm{MHz}$ ) $\delta: 3.79$ (s, 2H, $\left.-\mathrm{CH}_{2} \mathrm{CO}-\right), 4.61\left(\mathrm{~s}, 2 \mathrm{H},-\mathrm{CH}_{2} \mathrm{~S}-\right), 7.15(\mathrm{t}, 2 \mathrm{H}$, $J=8 \mathrm{~Hz}, \mathrm{H}_{2,6}$-4-fluorophenyl), 7.33 (t, 2H, $J=8 \mathrm{~Hz}$, $\mathrm{H}_{3,5}$-4-fluorophenyl), 7.61 (t, $1 \mathrm{H}, J=8 \mathrm{~Hz}, \mathrm{H}_{5}-3-$ nitrophenyl), 7.84 (d, $1 \mathrm{H}, J=8 \mathrm{~Hz}, \mathrm{H}_{6}$-3-nitrophenyl), 8.13 (d, $1 \mathrm{H}, J=8 \mathrm{~Hz}, \mathrm{H}_{4}$-3-nitrophenyl), 8.28 (s, $1 \mathrm{H}$, $\mathrm{H}_{2}$-3-nitrophenyl). IR ( $\left.\mathrm{KBr}, \mathrm{cm}^{-1}\right): 3443$ (stretch, $\mathrm{N}-\mathrm{H}$ ), 2922 (stretch, C-H, aromatic), 1693 (stretch, $\mathrm{C}=\mathrm{O}$ ), 1558 (bend, N-H), 1516, 1354, 1301, 1218, 1156, 1091, 836. MS (m/z, \%): $404(\mathrm{M}+, 45), 149$ (65), 145 (55), 136 (95), 109 (100), 97 (15), 83 (75), 69 (90), 55 (45), 43 (55).

2-(4-Fluorophenyl)-N-(5-(4-nitrobenzylthio)-1,3,4thiadiazol-2-yl)acetamide (3i); MP: $192^{\circ}$, yield: $85 \%,{ }^{1} \mathrm{HNMR}$ (DMSO-d, $\left.400 \mathrm{MHz}\right) \delta: 3.78$ (s, 2H, $\left.-\mathrm{CH}_{2} \mathrm{CO}-\right), 4.5$ (s, 2H, - $\mathrm{CH}_{2} \mathrm{~S}-$ ), 7.01 (t, $2 \mathrm{H}, J=8 \mathrm{~Hz}$, $\mathrm{H}_{2,6}$-4-fluorophenyl), $7.32\left(\mathrm{t}, 2 \mathrm{H}, J=8 \mathrm{~Hz}, \mathrm{H}_{3,5}-4-\right.$ fluorophenyl), 7.58 (d, $2 \mathrm{H}, J=8 \mathrm{~Hz}, \mathrm{H}_{2,6}$-4-nitrobenzyl), 8.14 (d, 2H, $J=8 \mathrm{~Hz}, \mathrm{H}_{3,5}$-4-nitrobenzyl), 12.62 (brs, $\mathrm{NH}$ ). IR $\left(\mathrm{KBr}, \mathrm{cm}^{-1}\right) \bar{v}: 3150$ (stretch, N-H), 3100 (stretch, C-H, aromatic), 2900 (stretch, C-H, aliphatic), 1693 (stretch, $\mathrm{C}=\mathrm{O}$ ), 1564 (bend, N-H), 1554, 1512, 1346, 1298, 1226, 1172, 1157, 1064, 960, 790. MS
( $m / z, \%): 404(\mathrm{M}+, 20), 149(60), 145$ (90), $136(75)$, 109 (100), 97 (35), 83 (55), 69 (90), 55 (80), 43 (85).

2-(4-Fluorophenyl)-N-(5-(3-methoxybenzylthio)1,3,4-thiadiazol-2-yl)acetamide (3j); MP: 172-175 , yield: $24 \%,{ }^{1} \mathrm{HNMR}$ (DMSO-d $\left.{ }_{6}, 400 \mathrm{MHz}\right) \delta: 2.8$ $\left(\mathrm{s}, 3 \mathrm{H},-\mathrm{OCH}_{3}\right), 3.78$ (s, 2H, $\left.-\mathrm{CH}_{2} \mathrm{CO}-\right), 4.41(\mathrm{~s}, 2 \mathrm{H}$, $\left.-\mathrm{CH}_{2} \mathrm{~S}-\right), 6.81(\mathrm{~m}, 2 \mathrm{H}, 3$-methoxybenzyl), $6.91(\mathrm{~m}, 2 \mathrm{H}$, 3-methoxybenzyl), 7.01 (m, 2H, $\mathrm{H}_{2,6}$-4-fluorophenyl), 7.33 (m, 2H, $\mathrm{H}_{3.5}$-4-fluorophenyl), 12.58 (brs, NH). IR $\left(\mathrm{KBr}, \mathrm{cm}^{-1}\right) \bar{v}: 3155$ (stretch, N-H), 2894 (stretch, C-H, aliphatic), 1700 (stretch, $\mathrm{C}=\mathrm{O}$ ), 1607, 1561 (bend, $\mathrm{N}-\mathrm{H}), 1488,1352,1298,1271,1156,1049,962,839$, 780. MS (m/z, \%): $389\left(\mathrm{M}^{+}, 90\right), 269$ (50), 121 (100), 109 (40).

2-(4-Fluorophenyl)-N-(5-(4-methoxybenzylthio)1,3,4-thiadiazol-2-yl)acetamide (3k); MP: $185-187^{\circ}$, yield: $44 \%,{ }^{1} \mathrm{HNMR}$ (DMSO-d $\left.{ }_{6}, 400 \mathrm{MHz}\right) \delta: 2.6$ $\left(\mathrm{s}, 3 \mathrm{H},-\mathrm{OCH}_{3}\right), 3.78$ (s, 2H, - $\left.\mathrm{CH}_{2} \mathrm{CO}-\right), 4.4(\mathrm{~s}, 2 \mathrm{H}$, $-\mathrm{CH}_{2} \mathrm{~S}-$ ), 6.82 (d, 2H, J=8 Hz, H${ }_{3,5}$-4-methoxybenzyl), 7.01 (t, 2H, $J=8 \mathrm{~Hz}, \mathrm{H}_{2,6}$-4-fluorophenyl), 7.28 (d, 2H, $J=8 \mathrm{~Hz}, \mathrm{H}_{2,6}-4$-methoxybenzyl), 7.33 (t, 2H, $J=8 \mathrm{~Hz}$, $\mathrm{H}_{3,5}$-4-fluorophenyl), 12.55 (brs, NH). IR ( $\left.\mathrm{KBr}, \mathrm{cm}^{-1}\right)$ ṽ: 3160 (stretch, N-H), 3040 (stretch, C-H, aromatic), 2900 (stretch, C-H, aliphatic), 2835 (stretch, C-H, aliphatic), 1689 (stretch, $\mathrm{C}=\mathrm{O}$ ), 1556 (bend, N-H), $1510,1420,1300,249,1168,1040,833$. MS ( $\mathrm{m} / z, \%)$ : 389 (M+, 80), 269 (35), 121 (100), 109 (85).

N-(5-(benzylthio)-1,3,4-thiadiazol-2-yl)-2-(4fluorophenyl)acetamide (31); MP: $188^{\circ}$, yield: $23 \%,{ }^{1} \mathrm{HNMR}$ (DMSO-d, $\left.400 \mathrm{MHz}\right) \delta: 3.80$ (s, - $\mathrm{CH}_{2}-$ CO-), 4.47 (s, - $\mathrm{CH}_{2} \mathrm{~S}-$ ), 7.15 (t, 2H, $J=8 \mathrm{~Hz}, \mathrm{H}_{2,6}-4-$ fluorophenyl), 7.24-7.33 (m, 5H, benzyl-S-), 7.38 (t, $2 \mathrm{H}, J=8 \mathrm{~Hz}, \mathrm{H}_{3,5}$-4-fluorophenyl), 12.86 (s, NH). IR $\left(\mathrm{KBr}, \mathrm{cm}^{-1}\right.$ ): 3140 (stretch, N-H), 3120 (stretch, C-H, aromatic), 2922 (stretch, C-H, aliphatic), 2806, 1693 (stretch, $\mathrm{C}=\mathrm{O}$ ), 1580 (bend, N-H), 1500, 1290, 1222, 1120, 1080, 790. MS (m/z, \%): $359\left(\mathrm{M}^{+1}, 95\right), 359$ (100), 223 (20), 181 (20), 148 (30), 109 (80), 91 (75).

\section{Docking studies:}

Molecular docking studies were performed using ArgusLab 4.0 software ${ }^{[22-25]}$. All intended ligands were constructed in ArgusLab workspace and energy minimization was performed for all ligands by AM1 as semiemperical method. The pdb file of c-Src protein tyrosine kinase in complex with dasatinib (pdb code: 3G5D) and abl tyrosine kinase in complex with imatinib (pdb code: $3 \mathrm{~K} 5 \mathrm{~V}$ ) were downloaded from Brookhaven protein databank ${ }^{[26]}$. The geometry 
optimization of each protein structures was performed using universal force field as a molecular mechanic method. The docking process was done for all ligands in the workspace of ArgusLab software after defining the related groups for each ligand and also for related protein. The binding location of dasatinib and imatinib were defined as binding site for finding the best pose and conformation for all ligands. Binding free energies were calculated and listed in Table 1. Binding mode and related interactions of ligands were explored in ArgusLab software.

\section{MTT assay:}

The MTT assay is based on the reduction by mitochondrial dehydrogenase in metabolically active cells of the novel tetrazolium compound, MTS, to the water-soluble formazan that absorbs at $490 \mathrm{~nm}$. Prostate carcinoma (PC3), neuroblastoma (SKNMC) and breast cancer (MCF-7) cells were seeded in each well of a 96-microwell plate and treated with various concentrations of the compound(s). Utilized cell lines were obtained from Pasteur Institute (Tehran, Iran). Cells were cultured in $37^{\circ}, \mathrm{CO}_{2}$ incubator $\left(5 \% \mathrm{CO}_{2}\right)$ in Dulbecco's modified Eagle's medium with $5 \%$ (v/v) fetal bovine serum, $100 \mathrm{U} / \mathrm{ml}$ penicillin and $100 \mathrm{mg} / \mathrm{ml}$ streptomycin and containing $10 \%$ heat-inactivated fetal bovine serum. $24 \mathrm{~h}$ after seeding cells were incubated overnight with different concentrations of the test compounds and then they were subjected to MTT assay for toxicity evaluation.

Cytotoxic effects of derivatives was measured using

TABLE 1: BINDING FREE ENERGIES OF THE COMPOUNDS 3a-3l AFTER DOCKING TO THE ACTIVE SITE OF ABL AND C-SRC TYROSINE KINASES

\begin{tabular}{lcccc}
\hline \multirow{2}{*}{ Compounds } & & \multicolumn{3}{c}{ Binding free energy $(\mathrm{Kcal} / \mathrm{mol})$} \\
\cline { 3 - 5 } & & $\mathrm{Abl}(\mathrm{A})$ & $\mathrm{c}-\mathrm{SrC}(\mathrm{A})$ & $\mathrm{C}-\mathrm{SrC}(\mathrm{B})$ \\
\hline & Imatinib & -9.28 & - & - \\
$\mathrm{3a}$ & Dasatinib & - & -8.44 & -8.77 \\
$3 \mathrm{~b}$ & $2-\mathrm{F}$ & -13.19 & -12.38 & -11.50 \\
$3 \mathrm{c}$ & $3-\mathrm{F}$ & -12.80 & -11.66 & -11.65 \\
$3 \mathrm{~d}$ & $4-\mathrm{F}$ & -13.72 & -11.60 & -10.80 \\
$3 \mathrm{e}$ & $2-\mathrm{Cl}$ & -13.69 & -11.92 & -11.65 \\
$3 \mathrm{f}$ & $3-\mathrm{Cl}$ & -14.11 & -12.65 & -12.01 \\
$3 \mathrm{~g}$ & $4-\mathrm{Cl}$ & -14.00 & -10.95 & -13.10 \\
$3 \mathrm{~h}$ & $2-\mathrm{NO}_{2}$ & -12.70 & -10.73 & -10.90 \\
$3 \mathrm{i}$ & $3-\mathrm{NO}_{2}$ & -12.18 & -11.77 & -11.42 \\
$3 \mathrm{j}$ & $4-\mathrm{NO}_{2}$ & -12.66 & -11.09 & -10.20 \\
$3 \mathrm{k}$ & $3-\mathrm{OCH}_{3}$ & -12.73 & -11.48 & -11.28 \\
$3 \mathrm{l}$ & $4-\mathrm{OCH}_{3}$ & -11.87 & -10.73 & -10.28 \\
\hline
\end{tabular}

the colorimetric assay in which live cells can reduce 3-(4,5-dimethylthiazol-2-yl)-2,5-diphenyltetrazolium bromide (MTT) to blue formazan crystals ${ }^{[27]}$. Cells were exposed to the compounds for $24 \mathrm{~h}$ and then they were incubated with MTT $(0.5 \mathrm{mg} / \mathrm{ml}$, Sigma-Aldrich) for $4.5 \mathrm{~h}$ at $37^{\circ}$. The wells were aspirated and DMSO was added to dissolve blue formazan crystals. After 5 min the plate was read in an ELx808 microplate reader (Biotek) at $570 \mathrm{~nm}$. Data were collected from 9 independent experiments. Some untreated cells underwent the above procedure and were chosen as controls. The following formula was used to determine the percentage of cell viability: percentage of cell viability $=(\mathrm{OD}$ test $/ \mathrm{OD}$ control $) \times 100 . \mathrm{IC}_{50}$ were calculated by plotting the $\log _{10}$ of the percentage of viability versus drug concentration.

\section{RESULTS AND DISCUSSION}

A new series of 1,3,4-thiadiazole-based compounds were synthesized via a click reaction. Addition of benzyl chlorides following a short hand shaking for a few seconds led to the formation of appropriate product. Target derivatives bearing electron withdrawing substituents such as fluorine and chlorine demonstrated higher yields in these series. In fact, electron receiving activity of these moieties facilitated the click reaction. Interestingly, higher yields were also afforded while these moieties introduced at positions ortho and para of the phenyl residue. Namely, a direct relationship was observed between the electron receiving properties of moiety and reaction yielding. Fluorinated derivatives with more electron negativity in comparison with chlorine containing congeners exhibited higher yields. On the other hands, methoxy moiety caused an unfavourable effect on yield of click reaction and a poor yield obtained especially for compound $3 \mathrm{j}$ with meta positioning of the phenyl ring. Electron donating feature of the methoxy group attenuated the reactivity of benzyl chloride derivatives and reduced the yield of click reaction at position meta significantly, whereas the obtained outcome at position para for methoxy group was the same as other moieties.

The results of docking studies were obtained according to the Table 1. Binding free energies for all ligands were calculated and listed. All ligands showed potent inhibitory potency against both $\mathrm{Abl}$ and $\mathrm{C}$-Src tyrosine kinases. Compound 3e with 3-chloro substitution showed the highest binding free energy $(-14.11 \mathrm{Kcal} /$ mol) for inhibition of Abl tyrosine kinase. There are three hydrogen bonding interactions with $\mathrm{Thr} 334$, 


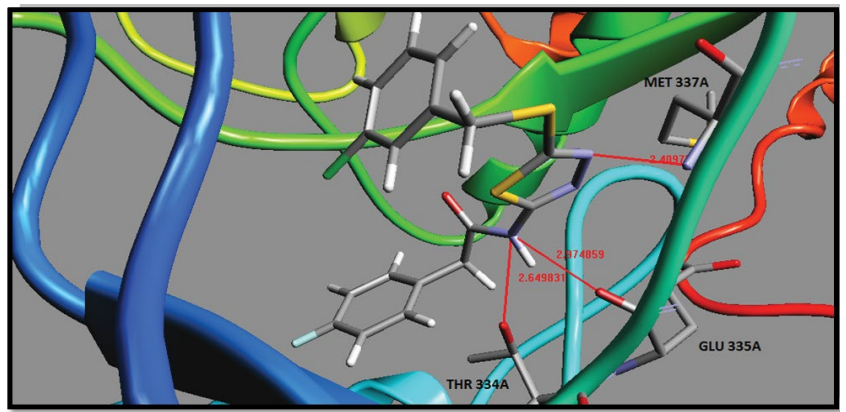

Fig. 3: Structure of compound $3 e$ in the active site of chain $A$ of Abl tyrosine kinase

Three hydrogen bonding interactions are observable with Met 337, Glu 335 and Thr 334. Nitrogen of 1,3,4-thiadiazole ring and amidic bond are responsible for these interactions

Met 337 and Glu 335 (fig. 3). This compound showed also higher in silico inhibitory potency in comparison with other compounds for inhibition of c-Src tyrosine kinase. The binding free energy for compound $3 \mathrm{e}$ is higher than imatinib and dasatinib. Lipophilicity and steric effects of the chlorine at this position may be the main logical reasons for this evident. Position 3 was the worst position for substitution of fluorine atom to inhibit the Abl tyrosine kinase. Substitution of fluorine at position 2 and 3 of the phenyl ring is suitable for inhibition of chain A and chain B of c-Src tyrosine kinase, respectively. On the other hand, fluorine moiety at position 4 demonstrated the lowest activity for both chain A and chain B of c-Src tyrosine kinase. Electron receiving property of the fluorine may be the cause of this phenomenon. Chlorine moiety at position 3 of the phenyl ring exhibited the best inhibitory activity against Abl tyrosine kinase. This position was also beneficial for inhibition of chain A as well as chain $\mathrm{B}$ of c-Src kinase. Chlorine caused better inhibition towards c-Src kinase compared to fluorine at position 3 . Position 2 was so detrimental for chlorine for in silico inhibition of Abl tyrosine kinase. The lowest inhibitory potency was observed for chlorine atom at positions 4 and 2 for inhibition of chain $\mathrm{A}$ and chain B of c-Src kinase, respectively. Nitro group with binding free energy equal to $-12.70 \mathrm{Kcal} / \mathrm{mol}$ at position 2 of the phenyl ring, showed the best activity toward inhibition of Abl tyrosine kinase. Nitro moiety exhibited the lowest binding free energy at position 3 for Abl, position 2 for chain A and position 4 for chain B of c-Src tyrosine kinase. Nitro moiety at position 3 demonstrated superior activity than fluorine at this position. Electrostatic interactions of the nitro group may potentiate the nitro interactions with active site. In the present study we investigated the methoxy substitution at positions 3 and 4. Methoxy moiety at position 3 afforded better inhibition than position 4 in both $\mathrm{Abl}$ and c-Src tyrosine kinase. Compound 31 without any moiety on the phenyl ring was also a potent inhibitor of $\mathrm{Abl}$ and c-Src tyrosine kinases compared to imatinib and dasatinib. Interestingly, this compound displayed higher activity than nitrated and methoxylated derivatives. It is likely that steric effect may impede the optimum interaction of the ligand with corresponding enzyme and therefore the removal of these groups enhanced the inhibitory potency. Fortunately, all of the docked compounds rendered remarkable potency in comparison with imatinib in Abl tyrosine kinase and also in c-Src tyrosine kinase.

All synthesized derivatives (3a-31) were tested against PC3, SKNMC and MCF-7 cell lines and obtained results were compared with imatinib as reference tyrosine kinase inhibitor drug (Table 2). Generally, tested derivatives showed higher cytotoxic activity against MCF-7 cell line. Chlorinated derivatives were the most effective compounds towards MCF-7 cell line in this series especially when chlorine atom substituted at positions ortho and meta. PC3 and SKNMC cell lines were highly resistant to the cytotoxicity effects of tested compounds. Fluorinated derivatives $(3 a, 3 b)$ demonstrated a remarkable and superior cytotoxic potency against MCF-7 cell line than imatinib except for 3c. It is probable that fluorine has effective interaction in the receptor while introduced on the positions ortho and meta. Movement of this moiety to the para position declined the effective interaction with its receptor. Chlorine substituent was also a beneficial group at position ortho as well as meta. It could be hypothesize that lipophilic and steric effects of the chlorine have important role in this observation. A reverse trend was observed about the nitro moiety

TABLE 2: CYTOTOXICITY $\quad\left(\mathrm{IC}_{50}, \mu \mathrm{M}\right) \quad$ OF COMPOUNDS 3a-3I

\begin{tabular}{lcccc}
\hline Compounds & $\mathbf{R}$ & PC3 & SKNMC & MCF-7 \\
\hline $3 \mathrm{a}$ & $2-\mathrm{F}$ & 83.12 & 95.58 & 26.94 \\
$3 \mathrm{~b}$ & $3-\mathrm{F}$ & 288 & 317 & 37.51 \\
$3 \mathrm{c}$ & $4-\mathrm{F}$ & 41.35 & 99.57 & 170.1 \\
$3 \mathrm{~d}$ & $2-\mathrm{Cl}$ & 47.95 & 156 & 4.43 \\
$3 \mathrm{e}$ & $3-\mathrm{Cl}$ & 129 & 72.61 & 4.13 \\
$3 \mathrm{f}$ & $4-\mathrm{Cl}$ & 148.8 & 123.2 & 14.85 \\
$3 \mathrm{~g}$ & $2-\mathrm{NO}_{2}$ & 154.2 & 133.9 & 61.31 \\
$3 \mathrm{~h}$ & $3-\mathrm{NO}_{2}$ & 288.8 & 122.4 & 93.94 \\
$3 \mathrm{i}$ & $4-\mathrm{NO}_{2}$ & 58.15 & 16.40 & 29.39 \\
$3 \mathrm{j}$ & $3-\mathrm{OCH}_{3}$ & 34.65 & 101.6 & 11.01 \\
$3 \mathrm{k}$ & $4-\mathrm{OCH}_{3}$ & 280 & 612.3 & 154.7 \\
$3 \mathrm{l}$ & $\mathrm{H}$ & 48.55 & 66.27 & 6.51 \\
Imatinib & - & 25.07 & 18.57 & 45 \\
\hline
\end{tabular}


in MCF-7 cell line. It means that nitro moiety caused a significant enhancement of the potency when substituted at position para. It is likely that hydrophilic property of the nitro group assist the molecule to interact more effective than lipophilic groups such as chlorine. This trend was also occurred in SKNMC cell line. Methoxy moiety increased the cytotoxic activity of the tested compound when inserted at position meta of the phenyl residue (compound 3j). Steric effect, moderate lipophilic property and finally hydrogen bonding activity are the most likely reason for cytotoxic activity. Utility of the phenyl residue without application of any substituent (compound 31) led to the favourable anticancer activity. Hydrophobic feature of the phenyl residue may responsible for this evident.

According to the obtained data from docking studies compounds 3a-31 may act as potent inhibitors of Abl as well as c-Src tyrosine kinase in comparison with imatinib. But, further experimental investigations such as enzymatic procedures are needed to prove it. MTT assay exhibited compounds $3 \mathrm{~d}$ and $3 \mathrm{e}$ can be potential anticancer candidates in breast cancer as seen towards MCF-7 cell line. Besides, a novel and facile approach was discovered for the synthesis of these compounds via click reaction.

\section{Acknowledgements:}

The current project was supported by research council of Kermanshah University of Medical Sciences financially. This work was performed in partial fulfillment of the requirement for PharmD of $\mathrm{Mr}$ Yazdan Bahmani (approved proposal No. 90043).

\section{Conflict of interest:}

None.

\section{REFERENCES}

1. Bhuran HA, Kini SJ. Synthesis, anticancer activity and docking of some substituted benzothiazoles as tyrosine kinase inhibitors. J Mol Graph Model 2010;29:32-7.

2. Madhusudan S, Ganesan TS. Tyrosine kinase inhibitors in cancer therapy. Clin Biochem 2004;37:618-35.

3. Radi M, Crespan E, Botta G, Falchi F, Maga G, Manetti F, et al. Discovery and SAR of 1,3,4-thiadiazole derivatives as potent Abl tyrosine kinase inhibitors and cytodifferentiating agents. Bioorg Med Chem Lett 2008;18:1207-11.

4. Lv PC, Li HQ, Sun J, Zhou Y, Zhu HL. Synthesis and biological evaluation of pyrazole derivatives containing thiourea skeleton as anticancer agents. Bioorg Med Chem 2010;18:4606-14.

5. Park BS, El-Deeb IM, Yoo KH, Oh CH, Cho SJ, Han DK, et al. Design, synthesis and biological evaluation of new potent and highly selective ROS1-tyrosine kinase inhibitor. Bioorg Med Chem Lett 2009;19:4720-3.

6. van Erp NP, Gelderblom H, Guchelaar HJ. Clinical pharmacokinetics of tyrosine kinase inhibitors. Cancer Treat Rev 2009;35:692-706.

7. Araujo J, Logothetis C. Dasatinib: A potent SRC inhibitor in clinical development for the treatment of solid tumors. Cancer Treat Rev 2010;36:492-500.

8. Manley PW, Cowan-Jacob SW, Buchdunger E, Fabbro D, Fendrich G, Furet P, et al. Imatinib: a selective tyrosine kinase inhibitor. Eur J Cancer 2002;38:S19-S27.

9. Mishra G, Singh AK, Jyoti K. Review article on 1, 3, 4-Thiadiazole derivatives and its Pharmacological activities. Int J ChemTech Res 2011;3:1380-93.

10. Kalidhar U, Kaur A. 1,3,4-Thiadiazole Derivatives and their Biological Activities: A Review. Res J Pharm Biol Chem Sci 2011;4:1091-106.

11. Siddiqui N, Ahuja P, Ahsan W, Pandeya SN, Alam MS. Thiadiazoles: Progress Report on Biological Activities. J Chem Pharm Res 2009;1:19-30.

12. Singh AK, Mishra G, Jyoti K. Review on Biological Activities of 1,3,4-Thiadiazole Derivatives. J Appl Pharm Sci 2011;5:449.

13. Kumar D, Vaddula BR, Chang KH, Shah K. One-pot synthesis and anticancer studies of 2-arylamino-5-aryl-1,3,4thiadiazoles. Bioorg Med Chem Lett 2011;21:2320-3.

14. Farshori NN, Banday RM, Ahmad A, Khan AU, Rauf A. Synthesis, characterization, and in vitro antimicrobial activities of 5-alkenyl/hydroxyalkenyl-2-phenylamine1,3,4-oxadiazoles and thiadiazoles. Bioorg Med Chem Lett 2010;20:1933-8.

15. Matysiak J, Opolski A. Synthesis and antiproliferative activity of N-substituted 2-amino-5-(2,4-dihydroxyphenyl)-1,3,4thiadiazoles. Bioorg Med Chem 2006;14:4483-9.

16. Ibrahim DA. Synthesis and biological evaluation of 3,6-disubstituted $\quad[1,2,4]$ triazolo[3,4-b][1,3,4]thiadiazole derivatives as a novel class of potential anti-tumor agents. Eur J Med Chem 2009;44:2776-81.

17. Supuran CT, Scozzafava A. Carbonic anhydrase inhibitors - Part 94. 1,3,4-Thiadiazole-2-sulfonamide derivatives as antitumor agents. Eur J Med Chem 2000;35:867-74.

18. Aliabadi A, Eghbalian E, Kiani A. Synthesis and cytotoxicity evaluation of a series of 1,3,4-thiadiazole based compounds as anticancer agents. Iran J Basic Med Sci 2013;11:1133-8.

19. Mohammadi-Farani A, Heidarian N, Aliabadi A. N-(5Mercapto-1,3,4-thiadiazol-2-yl)-2-phenylacetamide derivatives: Synthesis and in vitro cytotoxcity evaluation as potential anticancer agents. Iran J Pharm Res 2014;12:487-92.

20. Aliabadi A, Hasanvand Z, Kiani A, Mirabdali SS. Synthesis and in vitro cytotoxicity assessment of $\mathrm{N}-(5-($ Benzylthio)1,3,4-thiadiazol-2-yl)-2-(4-(trifluoromethyl)phenyl) acetamide with potential anticancer activity. Iran J Pharm Res 2013;4:687-93.

21. Aliabadi A, Shamsa F, Ostad SN, Emami S, Shafiee A, Davoodi J, et al. Synthesis and biological evaluation of 2-Phenylthiazole-4-carboxamide derivatives as anticancer agents. Eur J Med Chem 2010;11:5384-9.

22. ArgusLab, a molecular modeling, graphics, and drug design program for Windows operating systems. Available from: http://www.arguslab.com/arguslab.com/ArgusLab.html.

23. Fernandez D, Aviles FX, Vendrell J. A new type 
of five-membered heterocyclic inhibitors of basic metallocarboxypeptidases. Eur J Med Chem 2009;44:326671.

24. Mohammadi-Farani A, Ahmadi A, Nadri H, Aliabadi A. Synthesis, docking and acetylcholinesterase inhibitory assessment of 2-(2-(4-Benzylpiperazin-1-yl)ethyl) isoindoline-1,3-dione with potential anti-alzheimer effects. Daru 2013;21:47-55.

25. Aliabadi A, Foroumadi A, Safavi M, Kaboudanian Ardestani S. Synthesis, cytotoxicity assessment, and molecular docking of 4-Substituted-2-p-thiazole derivatives as probable c-Src and erb tyrosine kinase inhibitors. Croat Chem Acta 2013;86:245-51.

26. Protein Data Bank, A Structural View of Biology. Available from: http://RCSB.org.

27. Mohammadi-Farani A, Ghazi-Khansari M. Sahebgharani M. Glucose concentration in culture medium affects mRNA expression of TRPV1 and CB1 receptors and changes capsaicin toxicity in PC12 cells. Iran J Basic Med Sci 2014;9:673-8. 